\title{
Die kriminelle Gesellschaft als mediale Konstruktion? Mediennutzung, Kriminalitätswahrnehmung und Einstellung zum Strafen
}

\author{
Von Michael Windzio und Matthias Kleimann
}

Zusammenfassung: Kriminalität ist ein Thema mit hoher öffentlicher Aufmerksamkeit. In den Massenmedien, insbesondere im Privatfernsehen, wird in den verschiedensten Formaten über Kriminalität berichtet. Interessanterweise glaubt der überwiegende Teil der Bevölkerung, die Kriminalität hätte in den letzten 10 Jahren deutlich zugenommen - eine Auffassung, die zumindest durch die Hellfelddaten der Polizeilichen Kriminalstatistik nicht gestützt wird.

Zugleich sind Einstellungen zum Strafen verbreitet, denen zufolge die von den Gerichten üblicherweise verhängten Strafen als zu gering angesehen werden.

In dieser Arbeit wird gezeigt, dass das Thema Kriminalität vor allem in Nachrichtensendungen des Privatfernsehens präsent ist und gerade jene Personen, die private Nachrichtensendungen nutzen, irrtümlicherweise davon ausgehen, die Kriminalität habe in den letzten 10 Jahren deutlich zugenommen.

Diese Fehleinschätzung bleibt wiederum nicht ohne Folgen, denn je stärker diese bei einer Person ausgeprägt ist, desto häufiger vertritt sie auch die Auffassung, die von den Gerichten verhängten Strafen seien zu milde - woraus sich möglicherweise Folgen für die Kriminalpolitik ergeben könnten.

\section{Einleitung}

Darstellungen von Kriminalität sind in den Massenmedien überaus präsent und treffen beim Rezipienten auf hohe Aufmerksamkeit. So ist das bereits Mitte des 19. Jahrhunderts entstandene Genre des Kriminalromans heutzutage aus den Angebotspaletten der Verlage nicht mehr wegzudenken und sichert einen nennenswerten Anteil der Umsätze. In fiktionalen Darstellungen entfaltet das Thema der Kriminalität seinen Reiz vor dem Hintergrund eher realistischer Handlungsumgebungen, die dem Beobachter nahe legen, dass eine Geschichte sich zumindest so abgespielt haben könnte.

Ein Realitätsanspruch ist non-fiktiven Formaten natürlicherweise inhärent und Realitätsnähe wird vor allem von bildlichen Darstellungen in Fernsehnachrichten erwartet. Vergleicht man jedoch, wie unterschiedlich verschiedene Nachrichtensendungen über ein und dasselbe Verbrechen berichten, wird deutlich, dass die Medien zwar einerseits Themen aus der Umwelt aufgreifen, andererseits aber sowohl die Themenwahl als auch die Form der Darstellung selbst bestimmen.

In dieser Arbeit werden die Wirkungen untersucht, die Kriminalitätsberichte in Nachrichtensendungen auf die Rezipienten haben. Geprüft wird, ob durch Nutzung von Nachrichten der für Kriminalitätsberichte besonders sensiblen privaten Fernsehsender die von der Bevölkerung vermutete Kriminalitätsentwicklung beeinflusst wird und ob damit eine rigidere Einstellung zum Strafen in Verbindung steht.

Ausgangspunkt der Untersuchung ist die Annahme, dass gerade bei schweren und seltenen Delikten, wie z.B. Banküberfall oder Mord, die Wahrscheinlichkeit, dass Personen unmittelbar Zeugen der Tat werden, überaus gering ist und persönliche Erfahrungen somit kaum zu einem Urteil über die Kriminalitätsentwicklung herangezogen werden können. Vielmehr wird die Mehrheit der Bevölkerung, die keinen unmittelbaren Zugang zu empirischen sozialwissenschaftlichen Analysen hat, erst durch die Massenmedien über Phänomene der gesellschaftli-

Soziale Welt 57 (2006), S. $193-215$ 
chen Makroebene informiert und muss ihre Einschätzung vor allem auf diese Informationsquelle stützen. Weil die Massenmedien jedoch über Kriminalität in höchst selektiver Weise berichten und vor allem private Fernsehsender gute Gründe haben, dem Thema Kriminalität große Aufmerksamkeit zu widmen, wird die Auffassung gefördert, Straftaten wären im Jahre 2003 viel bzw. sehr viel häufiger verübt worden als im Jahre 1993. Diese mediale Konstruktion der kriminellen Gesellschaft führt in der Bevölkerung also zu einer falschen Einschätzung der Kriminalitätsentwicklung. Ist dieser kognitive »Bias « einmal erzeugt, ist es nur konsequent, wenn auf Basis von Alltagstheorien über eine abschreckende Wirkung von Strafe eine härtere Sanktionspraxis gefordert wird, um die vermeintliche Zunahme der Kriminalität einzudämmen.

Im folgenden Abschnitt dieser Arbeit wird zunächst anhand einer Gegenüberstellung der realen Entwicklung und dem von der Bevölkerung vermuteten Trend der Straftaten die verzerrte Wahrnehmung der Kriminalität empirisch nachgezeichnet. Der dritte Abschnitt dient einer systemtheoretischen Begründung der selektiven Kommunikation von Kriminalität durch die Massenmedien. Ausgehend von dieser Begründung sowie von einer modifizierten Version des Kultivationsansatzes George Gerbners werden anschließend Hypothesen über die Wirkung von Kriminalitätsdarstellungen in den Massenmedien auf die Rezipienten formuliert (Abschnitt 4). Nachdem in Abschnitt 5 die verwendeten Daten und Methoden beschrieben werden, soll im ersten Teil von Abschnitt 6 auf Basis wöchentlicher Zeitreihendaten von Fernsehnachrichten der Jahre 2000 bis 2003 gezeigt werden, dass es vor allem Nachrichtensendungen im privaten Fernsehen sind, in denen häufig über Kriminalität berichtet wird. Daraufhin wird anhand von Daten einer Bevölkerungsbefragung ein an Gerbners Ansatz orientiertes empirisches Modell spezifiziert, in dem der Effekt der Nutzung von Fernsehnachrichten privater Sender auf die wahrgenommene Kriminalitätsentwicklung sowie auf die Einstellung zur Strafzumessung untersucht wird.

\section{Die Entwicklung der Kriminalität aus Sicht der Bevölkerung}

Entgegen der weit verbreiteten Auffassung deutet sich in der Entwicklung der Polizeilichen Kriminalstatistik (PKS) zwischen 1993 und 2003 ein Rückgang der Straftaten an. Wie in Tabelle $1 \mathrm{zu}$ sehen ist, gab es in diesem Zeitraum nur bei Betrugsdelikten, Körperverletzung und Drogenhandel einen deutlichen Zuwachs, während die Zahlen in den meisten anderen Deliktbereichen abgenommen haben. Es wird allerdings vermutet, dass im Zuge spektakulärer Vorfälle an Schulen im Jahr 2003 die Anzeigebereitschaft der Körperverletzung gestiegen ist und dadurch ein Teil des Dunkelfeldes ins Hellfeld überführt wurde (Niedersächsisches Ministerium für Inneres und Sport 2005, S.15). ${ }^{1}$ Demgegenüber entspricht die in der PKS dokumentierte Zunahme von Betrugsdelikten einem realen Anstieg, da sich im Zuge der Vernetzung von Computern, vor allem aber durch Internetauktionen neue Gelegenheiten für Betrugsdelikte ergeben haben (ebd., S.12). Eine weitere Ausnahme stellt der laut PKS gestiegene Drogenhandel dar, bei dem es sich um ein so genanntes »Kontrolldelikt« handelt, ein Delikt also, bei dem die PKS besonders sensitiv für das Kontrollverhalten der Polizei ist.

Dem in der Tendenz ansonsten insgesamt abnehmenden Trend steht ein von der Bevölkerung vermuteter Anstieg der Kriminalität gegenüber. Während die Zahl der Delikte um 2,6 Prozent geringfügig abgenommen hat, gehen 27 Prozent der Befragten davon aus, Straftaten wären im Jahr 2003 sehr viel häufiger, 39 Prozent meinen, sie wären viel häufiger und 25 Prozent vermuten, sie wären etwas häufiger aufgetreten als im Jahr 1993. Die univariaten Verteilungen sind derart rechtsschief, dass aufgrund geringer Besetzungen die Kategorien »etwas seltener«, »viel seltener « und »sehr viel seltener« für die weitergehenden empirischen Analysen zusammengefasst wurden.

1) Durch die Realisierung eines Erlasses in Niedersachsen vom 30.09.2003 werden nun in der PKS alle Straftaten mit Schulbezug erfasst (ebd., S. 16). 
Tabelle 1: Einschätzung der Entwicklung ausgewählter Delikte für den Zehnjahreszeitraum 1993 bis 2003 in Prozent der Befragten

\begin{tabular}{|c|c|c|c|c|c|c|c|c|c|c|c|}
\hline & \multicolumn{3}{|c|}{ reale Entwicklung } & \multicolumn{7}{|c|}{ Vermutung der Befragten: } & \multirow[b]{2}{*}{$\mathrm{N}$} \\
\hline Delikt & $\begin{array}{l}\text { PKS } \\
1993\end{array}$ & $\begin{array}{l}\text { PKS } \\
2003\end{array}$ & $\Delta \%$ & $\begin{array}{l}\text { sehr } \\
\text { viel } \\
\text { häu- } \\
\text { figer }\end{array}$ & $\begin{array}{l}\text { viel } \\
\text { häu- } \\
\text { figer }\end{array}$ & $\begin{array}{l}\text { et- } \\
\text { was } \\
\text { häu- } \\
\text { figer }\end{array}$ & $\begin{array}{l}\text { gleich } \\
\text { ge- } \\
\text { blie- } \\
\text { ben }\end{array}$ & $\begin{array}{l}\text { et- } \\
\text { was } \\
\text { sel- } \\
\text { tener }\end{array}$ & $\begin{array}{l}\text { viel } \\
\text { sel- } \\
\text { tener }\end{array}$ & $\begin{array}{l}\text { sehr } \\
\text { viel } \\
\text { sel- } \\
\text { tener }\end{array}$ & \\
\hline Taten insgesamt & 6750613 & 6572135 & $-2,6 \%$ & $27 \%$ & $39 \%$ & $25 \%$ & $7 \%$ & $2 \%$ & $0 \%$ & $0 \%$ & 1977 \\
\hline Betrug & 419834 & 700013 & $+66,7 \%$ & $30 \%$ & $38 \%$ & $21 \%$ & $10 \%$ & $1 \%$ & $0 \%$ & $0 \%$ & 1980 \\
\hline Körperverletzung & 295005 & 467944 & $+58,6 \%$ & $26 \%$ & $36 \%$ & $25 \%$ & $11 \%$ & $2 \%$ & $0 \%$ & $0 \%$ & 1988 \\
\hline $\begin{array}{l}\text { Wohnungs- } \\
\text { einbruch }\end{array}$ & 227090 & 123280 & $-45,7 \%$ & $18 \%$ & $35 \%$ & $27 \%$ & $16 \%$ & $4 \%$ & $0 \%$ & $0 \%$ & 1989 \\
\hline Autodiebstahl & 214836 & 63240 & $-70,5 \%$ & $25 \%$ & $34 \%$ & $21 \%$ & $11 \%$ & $7 \%$ & $2 \%$ & $0 \%$ & 1982 \\
\hline Handtaschenraub & 7916 & 5986 & $-24,3 \%$ & $22 \%$ & $30 \%$ & $24 \%$ & $21 \%$ & $3 \%$ & $0 \%$ & $0 \%$ & 1985 \\
\hline vollend. Mord & 666 & 394 & $-40,8 \%$ & $16 \%$ & $24 \%$ & $27 \%$ & $28 \%$ & $5 \%$ & $1 \%$ & $0 \%$ & 1874 \\
\hline vollend. Sex.mord & 32 & 20 & $-37,5 \%$ & $19 \%$ & $26 \%$ & $28 \%$ & $23 \%$ & $4 \%$ & $1 \%$ & $0 \%$ & 1988 \\
\hline $\begin{array}{l}\text { Diebstahl } \\
\text { insgesamt }\end{array}$ & 4151087 & 3029390 & $-27,0 \%$ & $29 \%$ & $37 \%$ & $22 \%$ & $8 \%$ & $1 \%$ & $0 \%$ & $0 \%$ & 1980 \\
\hline Banküberfall & 1624 & 903 & $-44,4 \%$ & $13 \%$ & $22 \%$ & $25 \%$ & $29 \%$ & $10 \%$ & $2 \%$ & $0 \%$ & 1983 \\
\hline Drogenhandel & 37212 & 68701 & $+84,6 \%$ & $37 \%$ & $31 \%$ & $18 \%$ & $11 \%$ & $3 \%$ & $0 \%$ & $0 \%$ & 1977 \\
\hline Raubmord & 140 & & $-47,1 \%$ & $12 \%$ & $25 \%$ & $27 \%$ & $29 \%$ & $7 \%$ & $1 \%$ & $0 \%$ & 1977 \\
\hline sex. Miss. v. Kind. & 15430 & 15430 & $0 \%$ & $40 \%$ & $31 \%$ & $18 \%$ & $10 \%$ & $1 \%$ & $0 \%$ & $0 \%$ & 1844 \\
\hline
\end{tabular}

Die jeweilige Kriminalitätsbelastung einer Gesellschaft stellt ein Phänomen der sozialen Makroebene dar, über das Akteure ohne Zugang zu wissenschaftlich kontrolliert erhobenen Daten grundsätzlich keine sicheren Aussagen zu treffen vermögen. Möglicherweise haben einige der Befragten selbst - oder Kontaktpersonen innerhalb ihrer sozialen Netzwerke - die Erfahrung wiederholter Viktimisierung machen müssen. Doch gerade besonders schwere Delikte wie Tötung oder Banküberfall treten so selten auf, dass persönliche Erfahrungen nicht zu den in Tabelle 1 dargestellten Verteilungen führen können. Bei diesen selten auftretenden Delikten kann die Diskrepanz zwischen offizieller Statistik und Einschätzung durch die Bevölkerung auch nicht durch einen Unterschied zwischen Hell- und Dunkelfeld erklärt werden, da nicht davon auszugehen ist, dass die Mehrheit der Befragten durch persönliche Netzwerke über Opfer dieser Delikte erfahren hat. Angesichts dieser auffälligen Fehleinschätzungen stellt sich die Frage, auf Basis welcher Information die Bevölkerung zu Aussagen über derartige Trends gelangen kann. Geht man realistischerweise davon aus, dass der Großteil der Bevölkerung die offiziellen Statistiken nicht kennt, bleiben nur noch die Massenmedien als Quelle für Informationen über tatsächliche oder vermeintliche gesellschaftliche Entwicklungen. Dabei geht es vor allem um die Frage, wie häufig welches Medium in welcher Weise über Kriminalität berichtet. Das Verhältnis von Massenmedien, Rezipienten und Gesellschaft hat in der theoretischen Diskussion einen wichtigen Stellenwert und wird häufig in Verbindung mit der Konstruktion sozialer Wirklichkeit durch die Massenmedien diskutiert.

\section{Die Realität der Massenmedien}

In der Systemtheorie werden Massenmedien als beobachtende Systeme konzipiert (Luhmann 1996). Wie andere kognitive Systeme haben Massenmedien keinen erkennenden Zugang zu Realobjekten der Umwelt, der unabhängig von den Bedingungen der Erkenntnis selbst wäre. Während des momentanen Vollzugs eines Erkenntnisvorgangs kann das System nicht gleichzeitig seine eigenen kognitiven Strukturen, die »Frames«, reflektieren, mit denen es die Inputs der Umwelt verarbeitet. Wenn aber Systeme den Zugang zur Umwelt nur auf 
Grundlage der eigenen Frames erlangen können, folgt daraus zwangsläufig, dass deren Kommunikation über Umwelt nur auf eine in spezifischer Weise konstruierte Umwelt bezogen sein kann, die andere Systeme möglicherweise nicht teilen. Es sind daher die durch das System selbst getroffenen Unterscheidungen, anhand derer es die Umwelt abtastet und für welche die Umwelt keine unmittelbare Entsprechung bereithält (Luhmann 1996, S.172). In der Systemtheorie wird dieser Erkenntnisvorgang als operativer Konstruktivismus bezeichnet (ebd., S.163).

Innerhalb der Organisationen des Systems der Massenmedien wird unterschieden zwischen einer auf Themen der Kommunikation bezogenen Fremdreferenz und einer auf Funktionen der Kommunikation bezogenen Selbstreferenz. Selbstreferenz über die Funktion von Kommunikation, die etwa in einer Erhöhung der Einschaltquoten gesehen werden kann, eröffnet dem Beobachter gewisse Freiheitsgrade im Umgang mit der Realität. Das Weglassen bestimmter Informationen oder gar eine falsche Darstellung kann beispielsweise den Sensationswert der Kommunikation steigern, wenn man sich gegen das Risiko der Aufdeckung nur hinreichend absichert (Luhmann 1996, S.30). Daraus folgt aber weder, dass bewusst manipulative Praktiken dominieren, durch die Meinungen in der Bevölkerung einseitig geprägt werden, noch dass es keine Umwelt oder Wirklichkeit gäbe. An der Wirklichkeit können Organisationen, die massenmediale Kommunikationen erzeugen, durchaus scheitern, wenn etwa die von ihnen vorgenommen Selektionen nicht (mehr) mit den Selektionen des Publikums kompatibel sind (Carroll 1984).

Angeleitet durch den Code »Information/Nichtinformation« müssen die Massenmedien beständig Überraschendes - weil Neues - kommunizieren. Mit jeder Kommunikation entwerten sie bereits Bekanntes zu »Nichtinformation « und erzeugen auf diese Weise zugleich Ungewissheit und weiteren Informationsbedarf. Dadurch schaffen sie beständig Anlässe für rekursive Anschlusskommunikation und ermöglichen eine autopoietische Fortsetzung ihrer sinnhaften Operationen. Da das Mediensystem zudem nicht durch direkte Interaktion unter Anwesenden gestört wird, kann es, abgeschlossen von seiner Umwelt, ein hohes Maß an Eigenkomplexität aufbauen (Luhmann 1996, S.57). Welche Kriterien diesen vom System selbst vollzogenen Selektionen zu Grunde liegen, wurde in der empirischen Medienwissenschaft detailliert untersucht (Galtung/Ruge 1965; Peltu 1985; Plake 2004, S.110; Bonfadelli 2005b, S.48). ${ }^{2}$ So steigt der Überraschungswert einer Kommunikation durch markante Diskontinuität, indem mit bestehenden Erwartungen gebrochen wird (Luhmann 1996, S.61). Aufgrund ihrer immanenten Unsicherheit über den Ausgang sind überdies Konflikte als Themen gut geeignet. Besonderes Informationspotential haben außerdem Normverstöße, da sie explizit mit bestehenden Erwartungen brechen. Der Informationswert des Themas »Kriminalität« ist zudem dadurch gesichert, dass das eigene Sicherheitsgefühl des Beobachters durch non-fiktionale Darstellungen von Gewalt und Kriminalität in den Massenmedien beeinflusst ist (Reuband 1998). Darin liegt sicherlich einer der Gründe dafür, dass Selektionen der Massenmedien beim Rezipienten Aufmerksamkeit finden. Aufmerksamkeit wird auch bei psychischen Systemen durch Erwartungen strukturiert, also durch »(...) eine Orientierungsform, mit der das System die Kontingenz seiner Umwelt in Beziehung auf sich selbst abtastet und als eigene Ungewissheit in den Prozess autopoietischer Reproduktion übernimmt « (Luhmann 1984, S.362). Evolutionsbiologisch gesehen war es lange Zeit funktional, dass antizipative kognitive Schemata das Sehen steuerten und so aktiv bestimmte Information annah-

2) Das auch die Kontinuität von Sachverhalten ein Nachrichtenfaktor darstellen kann (Galtung/Ruge 1965), bedeutet, dass die ständige Wiederholung einer Nachricht den Rezipienten durch deren Bedeutsamkeit überrascht. In einem ähnlichen Sinn überrascht die Kontinuität von Nachrichten z.B. über Jugendgewalt in Pariser Vororten durch die scheinbar neue Qualität eines bereits bekannten sozialen Problems. 
men und andere ausblendeten (Neisser 1979, S.51). Die Aufmerksamkeit musste besonders sensibilisiert auf Sachverhalte gerichtet sein, die mit der eigenen physischen Existenz in Verbindung standen (Riedl 1988, S.112; Meyer 1982, S.30) oder zumindest die eigenen Interessen nachhaltig berührten. In diesem Sinne sind auch heute noch Informationen über antisoziale, kriminelle und gewaltbezogene Delikte extrem reizgeladen und finden besondere Beachtung sowohl beim Rezipienten als auch bei den Massenmedien - wenngleich es sich eben nur um Selektionen handelt, die das jeweilige System vornimmt und die keine Entsprechung in der Umwelt haben müssen.

Inhaltsanalysen der Lokalteile von Tageszeitungen haben in diesem Zusammenhang gezeigt, dass »(...) die Kurzberichterstattung der untersuchten Zeitungen ein Bild von Kriminalität vermittelt, das stärker von Gewalt und schweren Delikten geprägt ist, als dies der Realität entspricht « (Schönhagen/Brosius 2004, S.272). Man kann davon ausgehen, dass dies in ähnlicher Weise auch für Fernsehberichterstattung zutrifft. Es ist also sowohl theoretisch begründet als auch ein empirisch erhärteter Befund, dass Massenmedien erstens kein Abbild einer objektiven Realität liefern (können), und dass sie zweitens die »Erzeugung und Steuerung von Aufmerksamkeit« (Bonfadelli 2004a, S.231) des Publikums selbst leisten müssen. Letzteres gilt insbesondere im Kontext eines verschärften Wettbewerbs unter kommerziellen Fernsehanbietern. Besonders wichtig ist hier der seit Mitte der 1980er Jahre in der Bundesrepublik Deutschland flächendeckend erfolgte Ausbau des Privatfernsehens, infolgedessen Sender entstanden, die sich vollständig durch Werbeeinnahmen finanzieren und in noch stärkerem Maße als das öffentlich-rechtliche Fernsehen auf »quotenorientierte « Berichterstattung angewiesen sind. Mit dem Einstieg in das »Marktfernsehen « (im Gegensatz zum Gebührenfernsehen) »hatte generell die Unterhaltung an Bedeutung gewonnen, wobei auch solche Sendungen, die der Information und der Aufklärung dienen sollten, zusätzlich am Kriterium der Popularität, das heißt der Einschaltquote gemessen wurden« (Plake 2004, S.26).

So scheint das Thema »Kriminalität « mit seinem hohen Überraschungspotential insbesondere eine Angelegenheit privater Fernsehsender zu sein. Gegenwärtig werden immerhin 70 Prozent der Kriminalitätsberichterstattung im Kontext von Nachrichtensendungen allein von privaten Sendern geliefert (MedienTenor 11, 2004, S.33; vgl. Pfeiffer/Windzio/Kleimann 2004, S.421). Darstellungen von Kriminalität in non-fiktionalen Formaten der Fernsehsender sind zumeist nicht auf das engere regionale Umfeld begrenzt - weshalb sie unmittelbar bedeutsam werden für die Bildung kriminalpolitisch relevanter Meinungen. Inwieweit die von den politisch Verantwortlichen unterstellte öffentliche Meinung über Kriminalität und Strafen tatsächlich politische Entscheidungen beeinflusst, wie in der Literatur behauptet wird (Roberts 1992, S.101; Cavender 2004), lässt sich empirisch allerdings nicht ohne weiteres untersuchen. Aber auch unabhängig davon ist die Frage interessant, ob die vom Mediensystem beim Beobachten der Umwelt vorgenommenen Selektionen auch auf der Mikroebene, also auf der Seite der Rezipienten massenmedialer Kommunikation, ihre Entsprechung finden. So kann sich die Mehrheit der Wohnbevölkerung gerade bei eher selten auftretenden schweren Delikten nur vermittelt durch die »sekundäre« Wirklichkeit der Massenmedien über die Entwicklung der Kriminalität informieren (Reuband 1998, S.125). Variieren die in Abschnitt 2 dargelegten gravierenden Fehleinschätzungen über die Kriminalitätsentwicklung der Befragten systematisch mit Formen der Fernsehnutzung? Und gibt es einen Zusammenhang zwischen dem Ausmaß der Fehleinschätzung und dem Strafbedürfnis, also dem Verlangen nach härteren Strafen? 


\section{Theorien und Hypothesen der Medienwirkung}

Die aufgeworfenen Fragen nach dem Zusammenhang zwischen medialer Vermittlung gesellschaftlicher Kriminalität einerseits und Kriminalitätseinschätzung, Kriminalitätsfurcht und strafbezogenen Einstellungen in der Bevölkerung andererseits sind seit den 1970er Jahren immer wieder Forschungsgegenstand sozialwissenschaftlicher Studien gewesen (ein aktueller Überblick über die Forschung im angelsächsischen Raum bei Ditton et al. 2004). Ditton et al. kommen in einer Metaanalyse über den Zusammenhang von Kriminalitätsfurcht und Mediennutzung zu dem Ergebnis, dass sowohl für die Behauptung, es gäbe einen Zusammenhang, als auch für die Gegenthese eine Vielzahl von Belegen existiert, wobei die Zahl jener Studien etwas größer ist, die keinen Zusammenhang messen können. Eschholz (1997) kommt in ihrer Metaanalyse zu dem Schluss, dass in Phasen großen Medieninteresses an Kriminalitätsberichterstattung Effekte auf die Kriminalitätseinschätzung in der Bevölkerung auftreten können.

Eine große Zahl der Studien aus den 1970er und 1980er Jahren setzt sich dabei implizit oder explizit mit dem Kultivations-Ansatz der Forschungsgruppe um George Gerbner auseinander (eine recht aktuelle Darstellung dieses Ansatzes bei Signiorelli/Morgan 1990). Ein zentraler Untersuchungsgegenstand war von Beginn an die Wahrnehmung medialer Kriminalitätsdarstellungen und deren Wirkung auf die Rezipienten (perception of a mean and scary world). Gerbner konnte nachweisen, dass Rezipienten mit einem hohen Fernsehkonsum solche Bereiche von Kriminalität und Kriminalitätsbekämpfung überschätzten, die besonders häufig Gegenstand fiktionaler und non-fiktionaler Fernsehformate waren. Daraus resultierend wurde bei diesen Vielsehern auch eine höhere Kriminalitätsfurcht festgestellt. Der generelle Befund, dass Fernseh-Vielsehern eine maßgeblich durch Medien beeinflusste Wahrnehmung gesellschaftlicher Sachverhalte zu Eigen ist, wurde jedoch vielfach kritisiert und modifiziert (zur Kritik Hirsch 1980; Hirsch 1981). Dem Kultivations-Ansatz wurden vor allem methodische Mängel vorgeworfen. Neben der Argumentation, Gerbner mache mithilfe rein korrelativer Daten kausale Aussagen über Medienwirkungen, wurde immer wieder kritisiert, die Kultivationsforschung berücksichtige nicht genügend vermittelnde Variablen sowohl auf der Mediennutzungsseite (Rezipienten) wie auch auf der Seite des Medienangebotes. Trotzdem, so resümiert Bonfadelli (2004a), existieren zurzeit mehr als 300 empirische Studien, deren Ergebnisse (wenn auch meist mit kleinen Effekten) konsonant zu den Hypothesen der Kultivationsforschung sind (ebd., S.269).

Die neue Forschung hat Gerbners Fragestellung in vielerlei Hinsicht modifiziert und differenziert. Holbert, Shah und Kwak (2004) konnten zeigen, dass die Rezeption von Police Reality Shows bei Kontrolle verschiedener soziodemografischer Merkmale sowie der politischen und religiösen Ansichten der Befragten einen hoch signifikanten Einfluss auf Kriminalitätsfurcht und die Befürwortung härterer Strafen hat, während dies für die Rezeption fiktionaler Crime Dramas nicht bewiesen werden konnte. Für die Rezeption von Nachrichten fanden die Autoren signifikante, aber äußerst schwache Einflüsse. Lowry et al. (2003) kamen zu dem Schluss, dass die Kriminalitätsberichterstattung der großen amerikanischen TV-Networks zwischen 1978 und 1998 vier mal mehr Varianzaufklärung zur Wahrnehmung von Kriminalität als größtes gesellschaftliches Problem leistet als die staatliche Kriminalitätsstatistik. In Bezug auf die Wirkung von Kriminalitätsberichterstattung der Tagespresse kommt Eschholz (1997, S.50) zu dem Ergebnis, dass Wirkungen auf Kriminalitätseinschätzung und Kriminalitätsfurcht stark von der Art der journalistischen Darstellung abhängen. Dramatisierende Artikel mit hohem Neuigkeitswert und lokalem Bezug haben demnach die höchste Wahrscheinlichkeit, Kriminalitätseinschätzung und Kriminalitätsfurcht zu beeinflussen. Auf den deutschen Kontext bezogen bedeutet dies unter Umständen eine eher kleine Wirkungswahrscheinlichkeit von Zeitungsberichterstattung auf kriminalitätsbe- 
zogene Einschätzungen und Einstellungen. So konnte Derwein (1995; vgl. auch Scharf, Mühlenfeld und Stockmann 1999) zeigen, dass in der Kriminalitätsberichterstattung in deutschen Zeitungen zwar bestimmte Delikte (vor allem Gewaltdelikte) deutlich überrepräsentiert sind, dass aber selbst in Straßenverkaufszeitungen eine sachliche Art der Darstellung überwiegt.

Neben einer differenzierteren Forschung in Bezug auf Medium und Art der Kriminalitätsdarstellung fokussieren aktuelle Studien auch stärker auf moderierende und differenzierende Variablen auf Seiten der Rezipienten. Als Folge der wirksamen Ausschließung der Interaktion unter Anwesenden kommt es dazu, dass auch auf Seiten der Rezipienten eine Selektion vollzogen wird, die ihren eigenen Kriterien folgt (Luhmann 1996, S.12). Nach dem Paradigma des Uses-and-Gratification-Ansatzes stellen sich Medienrezipienten ihr Medienmenü speziell nach ihren eigenen Bedürfnissen zusammen (McQuail 1984; Rubin 2002). Dabei wählen sie nach der Mood-Management-Theorie solche Inhalte, mit denen sie ihre situativen, affektiven und kognitiven Bedürfnisse regulieren können. Andererseits bevorzugen sie nach konsistenz- bzw. dissonanztheoretischen Modellen solche Medieninhalte, die ihrer Erwartung nach eher mit ihrer Sicht und Einschätzung verschiedener Aspekte der Realität übereinstimmen. Vielfach wurde dieses Bild eines hochselektiven, bedürfnisorientierten Mediennutzers der gerbnerschen »Couch Potato«, die sich unreflektiert vom Angebot der Fernsehsender berieseln lässt, entgegengestellt. Vermeintliche Kultivationseffekte könnten, so die Kritik, vor allem daher rühren, dass eher ängstliche Menschen gerade solche Medienberichte nutzen und wahrnehmen, die ihre Einschätzung einer bedrohlichen Welt bestätigen. Demnach entwickeln also Menschen nicht eine andere Wahrnehmung der Kriminalität, weil sie extensiv bestimmte Medien nutzen, sondern sie nehmen besonders häufig bestimmte Medienangebote wahr, weil diese ihre Sicht der Welt bestätigen. Überdies bewegen sie sich aufgrund der größeren Kriminalitätsfurcht weniger außerhalb des eigenen Hauses und wenden sich deshalb häufiger dem Fernsehen zu als weniger ängstliche Personen (vgl. etwa van den Bulck 2004). Obwohl van den Bulck (2004) bei einem Vergleich der Kultivationshypothese mit diesen alternativen Erklärungsmustern eher empirische Belege für Kultivationseffekte fand, konnten andere Studien zeigen, dass die Faktoren Alter, Geschlecht, Bildung, Haushaltseinkommen, Wohnort, politische Einstellung und Religionszugehörigkeit einen Einfluss auf das individuelle Medienmenü, aber auch Einflüsse auf Kriminalitätswahrnehmung und kriminalitätsbezogene Einstellungen haben. So zeigt etwa Sotirovic (2001), dass Fähigkeit und Bereitschaft zu komplexem Denken mit der Bevorzugung komplexerer Medieninhalte und weniger extremen Strafhärtewünschen einhergehen.

Aus der Analyse des aktuellen Forschungsstandes ergeben sich folgende Implikationen für die weitere Forschung: Zum einen müssen sowohl auf Medien- als auch auf Rezipientenseite weitaus differenziertere Messungen vorgenommen werden als dies noch zu Beginn der Kultivationsforschung der Fall war. Medieninhalte müssen unterschieden, Medienrezeption muss als Produkt des sozialen Umfeldes und der persönlichen Prädispositionen und Bedürfnisse der jeweiligen Rezipienten konzeptionalisiert werden. Außerdem gilt es, den Komplex der verschiedenen Einflussvariablen auf kriminalitätsbezogene Wahrnehmungen und Einstellungen als ein Geflecht von Beziehungen dieser Variablen untereinander zu begreifen, die keinesfalls unabhängig voneinander zu betrachten sind. Zudem sollte es gelingen, die kriminalitätsbezogenen Wahrnehmungen und Einstellungen, also das Zusammenspiel von Kriminalitätswahrnehmung, Kriminalitätsfurcht und Einstellungen gegenüber der Sanktionierung von Kriminalität, zu beschreiben, ohne dabei soziale Einflüsse auf die Art der Mediennutzung zu vernachlässigen.

Um Aussagen über Medienwirkungen auf die Rezipienten treffen zu können, gilt es im ersten Schritt, die Medien selbst zu untersuchen. Im Mittelpunkt steht dabei zunächst die Frage, ob die Häufigkeit von Kriminalitätsberichten tatsächlich zwischen den unterschiedli- 
chen Nachrichtensendungen variiert. Wie erörtert, deuten bisherige Befunde darauf hin, dass private Sender aus ökonomischen Gründen stärker geneigt sind, über Kriminalität zu berichten, als öffentlich-rechtliche Sender. Die Selektionen des Mediensystems führen damit auf der Makroebene zu einer »operativ« konstruierten Wirklichkeit, auf die ein durchschnittlicher Rezipient jedoch angewiesen ist, wenn er sich über das Thema der Kriminalität informieren möchte.

Im zweiten Schritt der empirischen Untersuchung wird geprüft, ob sich Häufigkeit und Art der Mediennutzung dahingehend auswirken, dass entgegen der realen Entwicklung ein Anstieg, wenn nicht gar ein starker oder sehr starker Anstieg der Straftaten für die letzten zehn Jahre vermutet wird. Anschließend wird untersucht, inwieweit mit dem Ausmaß der selektiven Wahrnehmung auch das Strafbedürfnis der Rezipienten zunimmt.

Aus der bisherigen Argumentation lassen sich folgende Hypothesen herleiten: Wenn erstens die Massenmedien Freiheitsgrade in der Auswahl ihrer Themen nutzen können, wenn sie sich zweitens in ihrer Abhängigkeit von den Einschaltquoten unterscheiden, und wenn zum Dritten das Thema der Kriminalität eine vergleichsweise hohe Aufmerksamkeit des Rezipienten sichert, ist zu erwarten, dass die privaten Sender auch in non-fiktionalen Formaten wie Nachrichtensendungen zu einer erhöhten Berichterstattung über Kriminalität tendieren.

Hypothese 1: Die Zahl der Berichte über Kriminalität ist in privaten Nachrichtensendungen höher als in öffentlich-rechtlichen Nachrichtensendungen.

Folgt man dem oben ausgeführten Argument der Kultivation von Kriminalitätsfurcht und Kriminalitätswahrnehmung im Zuge der Mediennutzung, müsste die Kriminalitätsentwicklung umso stärker überschätzt werden, je häufiger die Rezipienten kriminalitätsbezogenen Sendungen privater Fernsehanstalten ausgesetzt sind. Und dieser Zusammenhang müsste ebenso auftreten, wenn der durch die Merkmale Alter, Bildungsniveau, Geschlecht und Erhebungsregion (Ost/West) bedingte selektive Zugang $\mathrm{zu}$ privaten Nachrichtensendungen kontrolliert ist (vgl. Abschnitt 6.2).

Hypothese 2: Je häufiger die Rezipienten Berichten über Kriminalität ausgesetzt sind, desto eher vertreten sie die Auffassung, die Kriminalität sei in den letzten 10 Jahren angestiegen.

Wird nun fälschlicherweise ein Anstieg der Kriminalität unterstellt, ist es kognitiv konsistent, auf der Grundlage von Alltagstheorien für härtere Strafen zu plädieren.

Hypothese 3: Je stärker Personen den Anstieg der Kriminalität überschätzen, desto eher ist die Meinung ausgeprägt, die von den Gerichten im Allgemeinen verhängten Strafen seien zu milde.

Im anschließenden Abschnitt werden die für die Prüfung dieser Hypothesen herangezogenen Daten und Methoden beschrieben.

\section{Daten und Methoden}

Wenngleich bereits einige empirische Hinweise dafür vorliegen, dass Nutzer des Privatfernsehens zumindest in quantitativer Hinsicht intensiver mit Berichten über Kriminalität konfrontiert werden, sind weitere Untersuchungen zu diesem Thema geboten. Das in Bonn ansässige Institut MedienTenor untersucht unter anderem täglich die wichtigsten Fernsehnachrichtensendungen, führt nach einem standardisierten Codierschema Zählungen der Berichte über bestimmte Themen durch und liefert darüber hinaus Einschätzungen über eher positive oder negative Beurteilungen von Akteuren oder Themen (vgl. MedienTenor 2004). Dazu gehören auch Berichte über Kriminalität, die teilweise wiederum differenziert nach Deliktsgruppen erfasst werden. In die folgenden Auswertungen dieser Daten gingen die 
Berichte über die Delikte insgesamt ein. In der Datei, die MedienTenor uns freundlicherweise bereitstellte, wurde die Kategorie »Terrorismus « explizit ausgeschlossen, da es sich - insbesondere seit dem 11. September 2001 - um eine neue und qualitativ andere Dimension von Kriminalität handelt. Im Zuge der Aufbereitung der Rohdaten von MedienTenor wurde für jede der sieben Sendungen eine Zeitreihe erstellt, die von Januar 2000 bis Dezember 2003 für jede Kalenderwoche die Anzahl der Berichte der sieben wichtigsten Nachrichtensendungen enthält. Die Abbildungen 1 und 2 zeigen die Verläufe der Inzidenzen für sieben Nachrichtensendungen über den genannten Zeitraum hinweg. Um die Frage zu beantworten, ob private Nachrichtensendungen signifikant häufiger über Kriminalität berichten als öffentlich-rechtliche Sendungen, muss berücksichtigt werden, dass nicht alle Sendungen gleichermaßen sensibel auf Themen ihrer Umwelt reagieren, dass also die mediale Präsenz bestimmter Ereignisse zwischen den Medien variieren kann.

Abbildung 1: Wöchentliche Anzahl der Berichte über Kriminalität, öffentlich-rechtliche Nachrichtensendungen

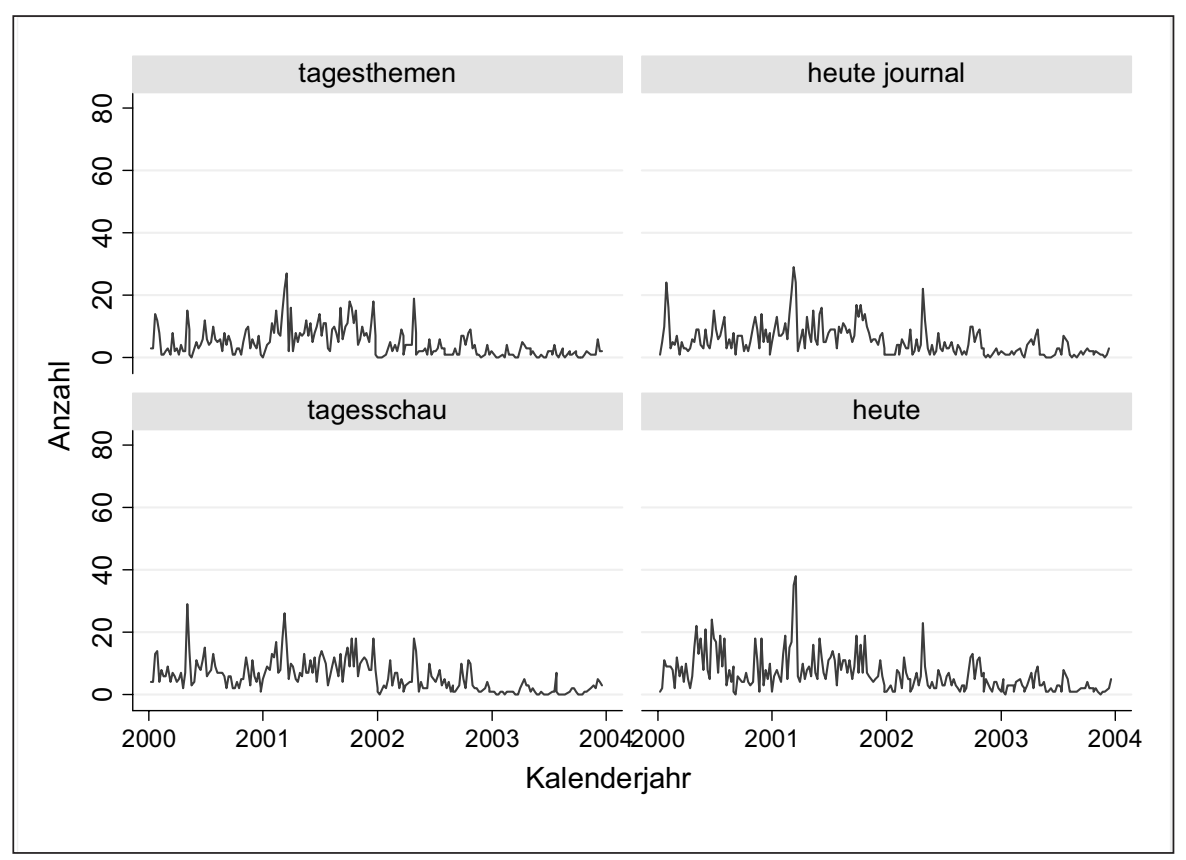


Abbildung 2: Wöchentliche Anzahl der Berichte über Kriminalität, private Nachrichten

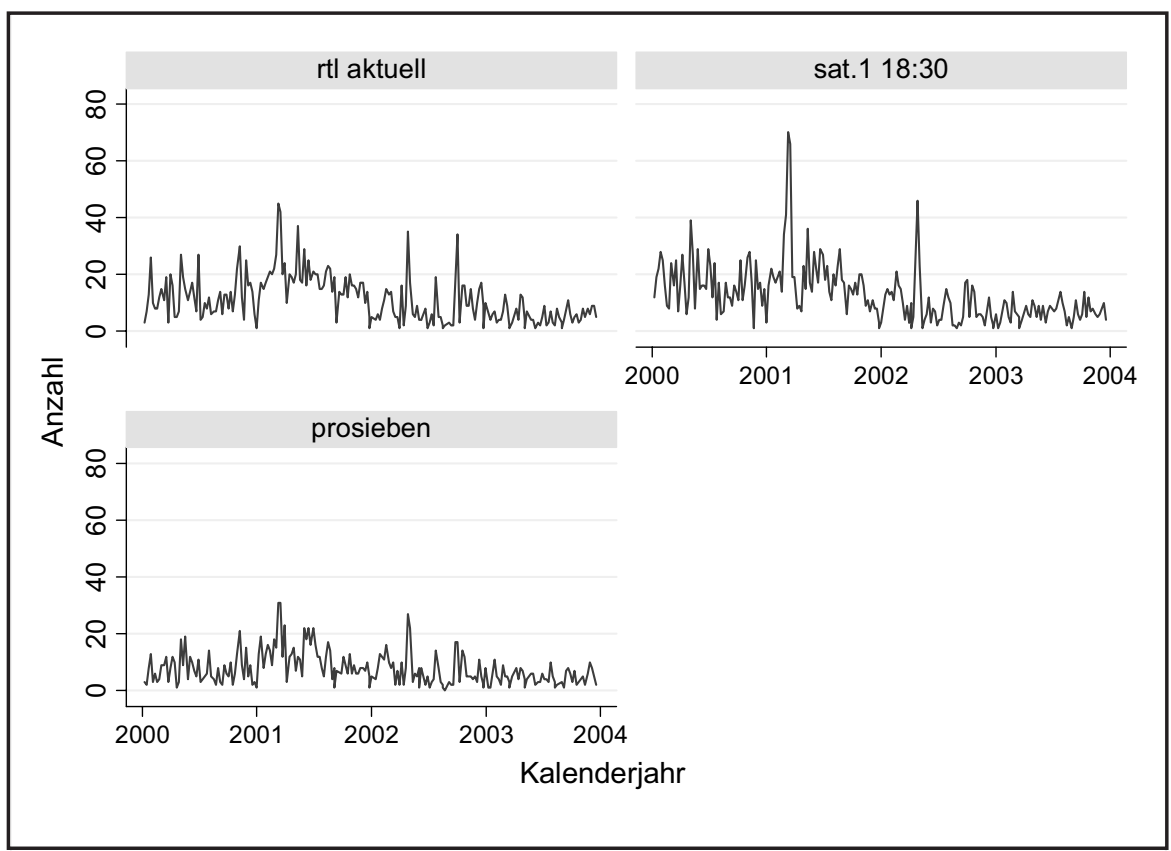

Quelle: MedienTenor Datenbank, eigene Berechnungen

Auf diese Weise können Ausreißer entstehen, wodurch die Anzahl der Berichte eines Senders insgesamt vergleichsweise hoch erscheint, obwohl eine Sendung sich, abgesehen von der kurzen Periode dieses Ereignisses, nicht von den anderen Sendungen unterscheidet. Abgesehen davon wäre es hilfreich zu wissen, ob die Zeitreihen einem Trend folgen und, sofern dies der Fall sein sollte, um welchen Trend es sich handelt.

Vorhergesagt wird die Anzahl der Berichte auf Basis eines Zähldatenmodells für Zeitreihendaten (STATA Corporation 2003, S.142). In diesem Modell wird zudem die (wöchentliche) Dauer der Sendungen kontrolliert, da diese Dauer als »risk exposure time « die strukturelle Gelegenheit für Kriminalitätsberichte vorgibt. Dabei variiert die wöchentliche Dauer je nach Sendung zwischen 75 und 185 Minuten. Der Logarithmus dieser Dauer wird als sogenannte offset-variable mit einem auf 1 fixierten Koeffizienten in die Zählgleichung aufgenommen (Long/Freese 2004, S.264).

Auf der Individualebene wird die Wirkung der Mediennutzung anhand ordinaler Regressionen geschätzt, die sowohl in der einfachen Form (Long 1997, S.120f) als auch in Form eines Pfadmodells mit latenten exogenen Variablen berechnet wurden (Muthén/ Muthén 1998-2004).

Die dem Modell zu Grunde liegenden Umfragedaten wurden Anfang Januar 2004 erhoben. Das Institut TNS Infratest wurde vom Kriminologischen Forschungsinstitut Niedersachsen beauftragt, eine Zufallsstichprobe von 2.000 Bundesbürgern postalisch zu befragen. Die Themen der Befragung waren unter anderem Kriminalität, Mediennutzung, persönliche Bedrohungsgefühle aufgrund von Kriminalität sowie das Ergreifen von Schutzmaßnahmen gegen Viktimisierung. Es wurde ferner ermittelt, aus welchen Quellen sich die Befragten über das Kriminalitätsgeschehen informieren. Die Fragen zu Mediennutzung, Kriminalitätswahrnehmung und Strafbedürfnis sind im Anhang dargestellt. 


\section{Ergebnisse}

\subsection{Makroebene: Kriminalitätsberichte im öffentlich-rechtlichen und privaten Fernse- hen}

In den Abbildungen 1 und 2 deutet sich bereits per Augenschein an, dass insgesamt die Zahl der kriminalitätsbezogenen Berichte in den Nachrichtensendungen des Privatfernsehens höher zu sein scheint, als in Sendungen des öffentlich-rechtlichen Fernsehens. Tabelle 2 enthält 6 Modellschätzungen auf Basis von Zähldatenmodellen, in denen dieser Befund statistisch getestet wurde.

Tabelle 2: Anzahl der Kriminalitätsberichte in Nachrichtensendungen, incidence rate ratios, negative Binomialregression für Zeitreihendaten

\begin{tabular}{lccccc|c}
\hline & Modell 1 & Modell 2 & Modell 3 & Modell 4 & Modell 5 & Modell 6 \\
\hline y (t - 1) & -- & -- & -- & -- & $1.015^{* *}$ & $1.033^{* *}$ \\
Zeit & -- & $4.397 * *$ & -- & $3.693^{* *}$ & $2.950^{* *}$ & $2.339^{* *}$ \\
Zeit $^{2}$ & -- & $0.406^{* *}$ & -- & $0.440^{* *}$ & $0.505^{* *}$ & $0.562^{* *}$ \\
Zeit $^{3}$ & -- & $1.134^{* *}$ & -- & $1.122^{* *}$ & $1.099^{* *}$ & $1.082^{* *}$ \\
Wochen 9-11, 2001 & -- & -- & $4.348^{* *}$ & $2.792^{* *}$ & $2.212^{* *}$ & $1.788^{* *}$ \\
Wochen 17-18, 2002 & -- & -- & $3.016^{* *}$ & $3.301^{* *}$ & $2.665^{* *}$ & $2.999^{* *}$ \\
Öffentlich-rechtl. TV & Referenz & Referenz & Referenz & Referenz & Referenz & Referenz \\
Privat TV & $2.115^{* *}$ & $1.990^{* *}$ & $2.137^{* *}$ & $1.998^{* *}$ & $1.855^{* *}$ & $2.409^{* *}$ \\
& & & & & & \\
\hline $\mathrm{R}$ & 10.20 & 10.79 & 10.64 & 11.54 & 13.15 & $\alpha=0.369^{*}$ \\
$\mathrm{~S}$ & 38.35 & 24.60 & 32.78 & 20.86 & 22.90 & pooled \\
\hline$\chi^{2}$ - (df) & $69.07(1)$ & $616.40(4)$ & $479.49(3)$ & $1118.85(6)$ & $1260.25(7)$ & $994.43(7)$ \\
Zahl der Beobachtun- & 1470 & 1470 & 1470 & 1470 & 1470 & 1470 \\
gen & 7 & 7 & 7 & 7 & 7 & 7 \\
Zahl der Sendungen & 7 & 7 & & & \\
\hline
\end{tabular}

* signifikant bei $5 \%$; ** signifikant bei $1 \%$; Zeit = Anzahl der Wochen seit Woche 1/2000

Modelle 1-5 mit zufälligem Effekt (STATA Corporation: 142)

Quelle: MedienTenor Datenbank, eigene Berechnungen

Tabelle 2 zeigt die Ergebnisse der sechs Modellschätzungen, in denen der Satz der erklärenden Variablen variiert wurde. Die Koeffizienten sind über den Ausdruck exp(b) in incidence rate ratios umgerechnet, die Änderungen im Verhältnis der gezählten Ereignisse anzeigen, wenn die erklärende Variable um eine Einheit zunimmt. Werte $<1$ bedeuten somit einen negativen, Werte $>1$ einen positiven Effekt. Dabei zeigt sich deutlich, dass der Effekt der Dummyvariable »Privatfernsehen « über alle Modelle recht robust ist. ${ }^{3}$

In den beiden Ausnahmeperioden, die insbesondere bei den Privatsendern, aber auch bei der Nachrichtensendung des ZDF (»heute«) zu Ausreißern führten, war die Zahl der Berichte

3) Mit N=7 Sendungen ist die Zahl der Gruppen für eine Spezifikation mit einem zufälligen Effekt sehr klein. Eine alternative Spezifikation als fixed effects-Modell lieferte ebenfalls das Ergebnis einer höheren Inzidenz bei Sendungen privater Fernsehanstalten. 
über Kriminalität gegenüber den anderen Perioden im Mittel um das 1,7fache bis 4,3fache erhöht. Zudem ist zu sehen, dass die mittlere Differenz zwischen den öffentlich-rechtlichen und den privaten Nachrichtensendungen nur in geringem Maße durch diese Ausnahmeperioden beeinflusst ist, da über alle vier Modelle hinweg die geschätzte Zahl der Berichte in Sendungen der privaten Fernsehanstalten ungefähr doppelt so hoch ist wie die der öffentlichrechtlichen. Hypothese 1 ist damit nicht widerlegt. Dieser einerseits sehr eindeutige, andererseits aber rein quantitative Befund stützt Resultate von Studien, die zeigen, dass Nachrichten privater Sender in der Tendenz emotionalisierender sind und Normabweichung als Information inszenieren (vgl. Plake 2004, S.104f).

Somit sprechen diese Befunde erstens dafür, dass es sich auf der Makroebene um eine selektive und damit konstruierte Wirklichkeit der Massenmedien handelt. Ansonsten würde der Stellenwert, der dem Thema der Kriminalität zugemessen wird, nicht zwischen privaten oder öffentlich-rechtlichen Nachrichtensendungen variieren. Zweitens hat sich erwartungsgemäß gezeigt, dass Nachrichtensendungen privater Fernsehanstalten auch unter Kontrolle von zeitlichen Trends sowie zweier Ausnahmeperioden ungefähr doppelt so häufig Kriminalität thematisieren wie öffentlich-rechtliche.

Abbildung 3: Trendanpassung Tagesthemen

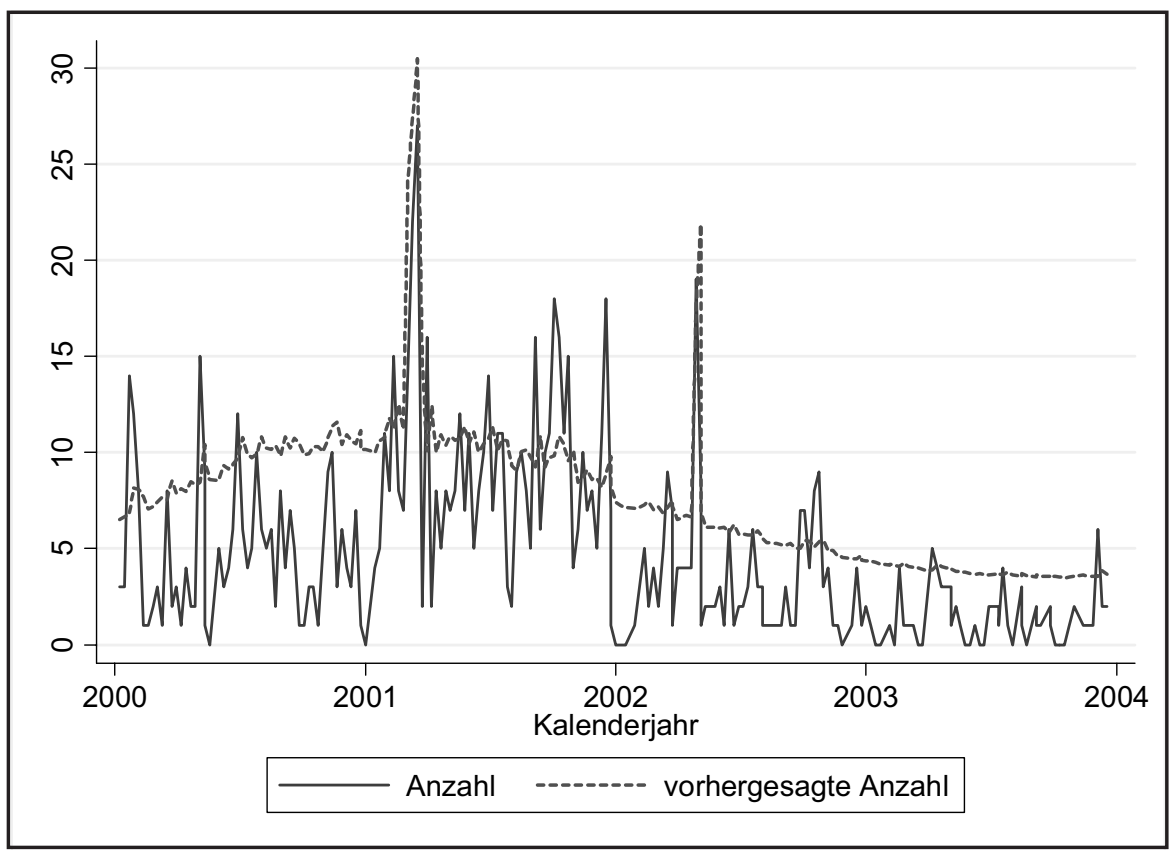

Quelle: MedienTenor Datenbank, eigene Berechnungen

In der Abbildung 3 sind exemplarisch anhand der »Tagesthemen« neben dem Trend auch die beiden als Ausreißer identifizierten Perioden abgebildet, die zu verzerrten Schätzungen hätten führen können, zumal insbesondere die »SAT1 18:30 Nachrichten« - als Sendung des Privatfernsehens - extreme Werte aufweisen (vgl. Abbildung 2). Es handelt sich daher dabei um die Wochen 9 bis 11 des Jahres 2001 sowie um die Wochen 17 bis 18 des Jahres 2002. Anfang März 2001 wurde die Leiche der 12-jährigen Schülerin Ulrike aus Eberswalde ge- 
funden. Sie wurde sexuell missbraucht und anschließend erwürgt. Die erste große Welle der Berichterstattung setzte nach dem Zeitpunkt des Verschwindens des Mädchens ein, thematisiert wurde vor allem die Suche nach Ulrike. Mit dem Fund der Leiche ab dem 9. März erreichte die Berichterstattung einen weiteren Höhepunkt, die Fahndung nach dem Täter, die Durchführung von und die Diskussion um Gentests standen nun im Vordergrund. Ende April, Anfang Mai des Jahres 2002 kam es zu der zweiten Ausreißerperiode, die ausgelöst wurde durch den Amoklauf eines Schülers an einem Erfurter Gymnasium.

Der in Abbildung 3 exemplarisch dargestellte Trend zeigt keinen monotonen Anstieg, sondern einen zunächst steigenden, dann abnehmenden Verlauf. Gegen Ende des Zeitfensters wird die Abnahme gebremst. Es kann daher nicht behauptet werden, dass zwischen den Jahren 2000 und 2004 die Berichte über Kriminalität zugenommen hätten. Die tendenzielle Abnahme seit dem Jahre 2002 könnte dadurch begründet sein, dass andere Themen wie der internationale Terrorismus und Krieg an Bedeutung gewonnen haben. Wichtig ist aber, dass die privaten Nachrichtensendungen mit ihrer größeren Affinität zum Thema Kriminalität seit den frühen 1990er Jahren zu einem festen Bestandteil der Medienlandschaft geworden sind und dadurch seit dieser Zeit die mediale Kommunikation über Kriminalität zugenommen hat.

Daraus ergibt sich unmittelbar die Frage, ob die selektive Beobachtung der Umwelt durch das Mediensystem Folgen für die von den Rezipienten vermutete Kriminalitätsentwicklung hat und - vermittelt über eine selektive Wahrnehmung auf Rezipientenseite - auch punitive Einstellungen, also das Verlangen nach härteren Strafen, fördert.

\subsection{Individualebene: Die Wirkung der Massenmedien auf Kriminalitätswahrnehmung und Strafbedürfnisse}

Die Frage nach den Wirkungen von massenmedialen Darstellungen von Kriminalität auf kriminalitätsbezogene Wahrnehmungen und Einstellungen bei den Rezipienten wurde in einer Vielzahl empirischer Studien untersucht. Wie in Abschnitt 4 dargelegt, sind die Befunde insgesamt eher heterogen: Obwohl sich in einigen Analysen deutliche Einflüsse abzeichnen, wie in der Studie von Pfeiffer, Windzio und Kleimann (2004), sind kausale Aussagen nicht immer eindeutig zu treffen. In der genannten Studie wurde gezeigt, dass insbesondere eine intensive Nutzung kriminalitätshaltiger Sendungen des Privatfernsehens eine Überschätzung der Kriminalitätsentwicklung fördert.

In der anschließenden empirischen Analyse wird dieser Befund nun detaillierter überprüft. Zunächst wird eine ordinale logistische Regression durchgeführt, um aus der geschätzten Gleichung die Relevanz des Mediennutzungseffektes auf die Wahrnehmung der Kriminalitätsentwicklung zu ermitteln. Als Prädiktoren dienen neben dem Alter der Befragten, ihrem Geschlecht und ihrem Bildungsniveau zwei latente Variablen, deren Faktorwerte in die Schätzung eingingen. In den Tabellen 3 und 4 sind die Faktorladungen der Items sowie die Reliabilität der Skalen aufgeführt. Es handelt sich dabei um die Konstrukte der Kriminalitätsfurcht - hier operationalisiert durch Vorsichtsmaßnahmen gegen Viktimisierung - und der Nutzungsskala »Privat TV «. 
Tabelle 3: Kriminalitätsfurcht: Vorsichtsmaßnahmen, Faktorladungen

\begin{tabular}{l|l}
\hline & \\
\hline $\begin{array}{l}\text { Ich verlasse das Haus bei Dunkel- } \\
\text { heit nur, wenn es unbedingt notwen- } \\
\text { dig ist }\end{array}$ & 0.54 \\
\hline $\begin{array}{l}\text { Ich meide bestimmte Straßen, Plätze } \\
\text { oder Parks }\end{array}$ & 0.77 \\
\hline $\begin{array}{l}\text { Ich weiche Fremden, denen ich im } \\
\text { Dunkeln begegne, nach Möglichkeit } \\
\text { aus }\end{array}$ & 0.76 \\
\hline $\begin{array}{l}\text { Ich vermeide es, abends öffentliche } \\
\text { Verkehrsmittel zu benutzen }\end{array}$ & 0.63 \\
\hline
\end{tabular}

Cronbachs alpha $=0.786$

Quelle: KFN-Befragung zu Kriminalität und

Strafen 2004, eigene Berechnungen

In Tabelle 5 sind die unstandardisierten Effekte auf die ordinale abhängige Variable der wahrgenommenen Kriminalitätsentwicklung dargestellt.
Tabelle 4: Skala »Nutzung kriminalitätshaltiger Nachrichten Privat $\mathrm{TV} \ll$ Faktorladungen

\begin{tabular}{l|l}
\hline & \\
\hline RTL Aktuell & 0.72 \\
18:30 - SAT 1 Nachrichten & 0.81 \\
Pro 7 Nachrichten & 0.72 \\
& \\
\hline
\end{tabular}

Cronbachs alpha $=0.82$

Quelle: KFN-Befragung zu Kriminalität und

Strafen 2004, eigene Berechnungen 
Tabelle 5: Rezeption von Kriminalitätsberichten in Nachrichtensendungen und vermutete Kriminalitätsentwicklung ${ }^{+}$

\begin{tabular}{l|l}
\hline \multicolumn{2}{l}{ unabhängige Variablen } \\
\hline$\tau 1$ & -3.21 \\
\hline$\tau 2$ & -1.69 \\
\hline$\tau 3$ & 0.15 \\
\hline$\tau 4$ & 2.01 \\
\hline Ausbildungsjahre & $-0.073^{* *}$ \\
\hline Alter (Jahre) & $0.012^{* *}$ \\
\hline Frau (=1, sonst 0) & $0.212^{*}$ \\
\hline & \\
\hline Privat TV (0-100\%) & $0.010^{* *}$ \\
\hline Kriminalitätsfurcht $(0-100 \%)$ & $0.012^{* *}$ \\
\hline Observations & 1554 \\
\hline$\chi^{2}$ (df) & $197.48(5)$ \\
\hline
\end{tabular}

+ ordinale logistische Regression, »Solche Straftaten sind nach meinem Eindruck in den letzten 10 Jahren 1=etwas/viel/sehr viel seltener; $2=$ gleich geblieben; $3=$ etwas häufiger; $4=$ viel häufiger; $5=$ =sehr viel häufiger geworden

brant test: $\chi^{2}-(\mathrm{df})=15.14(15), \mathrm{p}=0.442$

* signifikant bei $5 \%$; ** signifikant bei $1 \%$

Quelle: KFN-Befragung zu Kriminalität und Strafen 2004, eigene Berechnungen

Die beiden Faktorwerte der Skalen »Privat TV « und »Kriminalitätsfurcht« wurden so transformiert, dass jede Beobachtung den prozentualen Anteil am maximalen Wert der jeweiligen Skala zugewiesen bekam. Dies erleichtert die Interpretation der Effekte in Abbildung 4. Zu sehen ist in Tabelle 5, dass die Nutzung von kriminalitätshaltigen Sendungen im Privatfernsehen hoch signifikant die wahrgenommene Kriminalitätsentwicklung reduziert. Dies gilt auch unter Kontrolle von Ausbildung, Geschlecht, Alter und Kriminalitätsfurcht, wobei letztere einen geringfügig stärkeren Effekt aufweist. Anschaulicher sind vorhergesagte Wahrscheinlichkeiten der Befragten, sich in Abhängigkeit von ihrem Niveau der Mediennutzung in einer jeweiligen Kategorie der abhängigen Variablen zu befinden. Anhand der Regressionsgleichung aus Tabelle 5 wurde für Personen mit 11 Schulbildungsjahren (Mittelwert), einem mittleren Alter von 46 Jahren und einer mittleren Kriminalitätsfurcht (49 \% von $100 \%$ ) die Wahrscheinlichkeit für die jeweilige Kategorie vorhergesagt. 
Abbildung 4: Rezeption von Kriminalitätsberichten und vermutete Kriminalitätsentwicklung, konditionaler Effektplot

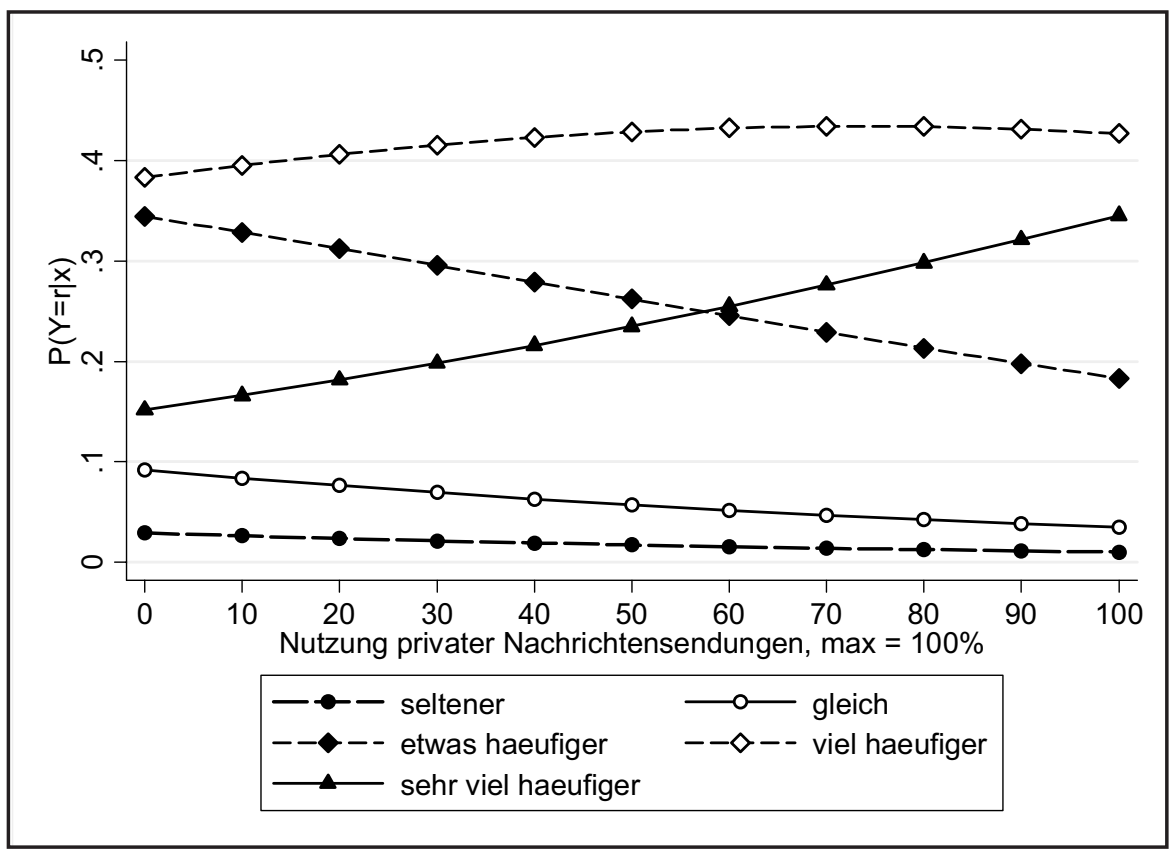

Quelle: KFN-Befragung zu Kriminalität und Strafen 2004, eigene Berechnungen

Der Verlauf der Kurven ist in Abbildung 4 folgendermaßen zu interpretieren: Der ohnehin sehr geringe Anteil von Personen, die eine Abnahme der Zahl der Delikte vermuten (»seltener «), nimmt mit steigender Nutzung privater Nachrichtensendungen ab. Ähnlich verhält es sich mit jenen, die von einer gleichgebliebenen Kriminalität ausgehen (»gleich«), deren Anteil von 7,6 Prozent auf 4,2 Prozent zurückgeht, wenn man jene mit einem Wert von 20 Prozent auf der Nutzungsskala »Privat TV « mit jenen vergleicht, die einen Wert von 80 Prozent aufweisen. Besonders deutlich zeigt sich die Relevanz des Effektes bei den Kategorien »etwas häufiger« und »sehr viel häufiger«. In Tabelle 1 wurde gezeigt, dass die Einschätzung der Entwicklung von Straftaten insgesamt eine stark rechtsschiefe Verteilung aufweist und die Befragten in hohem Maße zu der Auffassung tendieren, die Delikte wären im Jahr 2003 viel oder sehr viel häufiger aufgetreten. Jene Befragte, die einen Wert von Null auf der Nutzungsskala »Privat TV « aufweisen, meinen zu 34,4 Prozent, dass Straftaten 2003 gegenüber 1993 »etwas häufiger« aufgetreten wären. Dieser Wert nimmt auf 18,3 Prozent ab, wenn sie den Maximalwert auf der Nutzungsskala »Privat TV « erreichen. Komplementär dazu erhöht sich die Wahrscheinlichkeit der Kategorie »sehr viel häufiger « mit steigender Nutzung privater Fernsehnachrichten erheblich, nämlich von 15,1 Prozent beim Minimum bis auf 34,5 Prozent beim Maximum auf der Nutzungsskala »Privat TV «. Markante Differenzen finden sich aber auch bereits zwischen weniger extremen Gruppen, beispielsweise zwischen jenen mit 30 Prozent und jenen mit 70 Prozent auf der Nutzungsskala »Privat TV «: In der ersten Gruppe beträgt die Wahrscheinlichkeit der Kategorie »sehr viel häufiger« 19,8 Prozent, in der zweiten Gruppe 27,6 Prozent. 
Diese Vorhersagen liefern einen guten Eindruck von der quantitativen Bedeutsamkeit des Effektes. Ein naheliegender Einwand, der auch gegen Gerbners Kultivationsforschung formuliert wurde, könnte jedoch darin bestehen, dass man keinen direkten kausalen Einfluss der Nutzung privater Nachrichtensendungen auf die verzerrte Wahrnehmung der Kriminalitätsentwicklung unterstellen kann, weil auch die Neigung zu privaten Fernsehnachrichten sozial selektiv ist und unter Umständen von Faktoren abhängt, die sowohl mit der Affinität zum Privatfernsehen als auch mit der Kriminalitätswahrnehmung korrelieren. Demzufolge würde etwa das Bildungsniveau sowohl die Nutzungshäufigkeit privater Nachrichtensendungen als auch die Kriminalitätswahrnehmung beeinflussen. Zudem interessieren vor allem weitergehende Folgen, die eine übermäßige Präsenz der Kriminalität in den Massenmedien möglicherweise implizieren - und das könnte eine Zunahme rigider Einstellungen zum Strafen sein, die eine mangelnde Strafhärte von Politik und Justiz unterstellen.

Abbildung 5: Pfadmodell mit ordinalen abhängigen Variablen

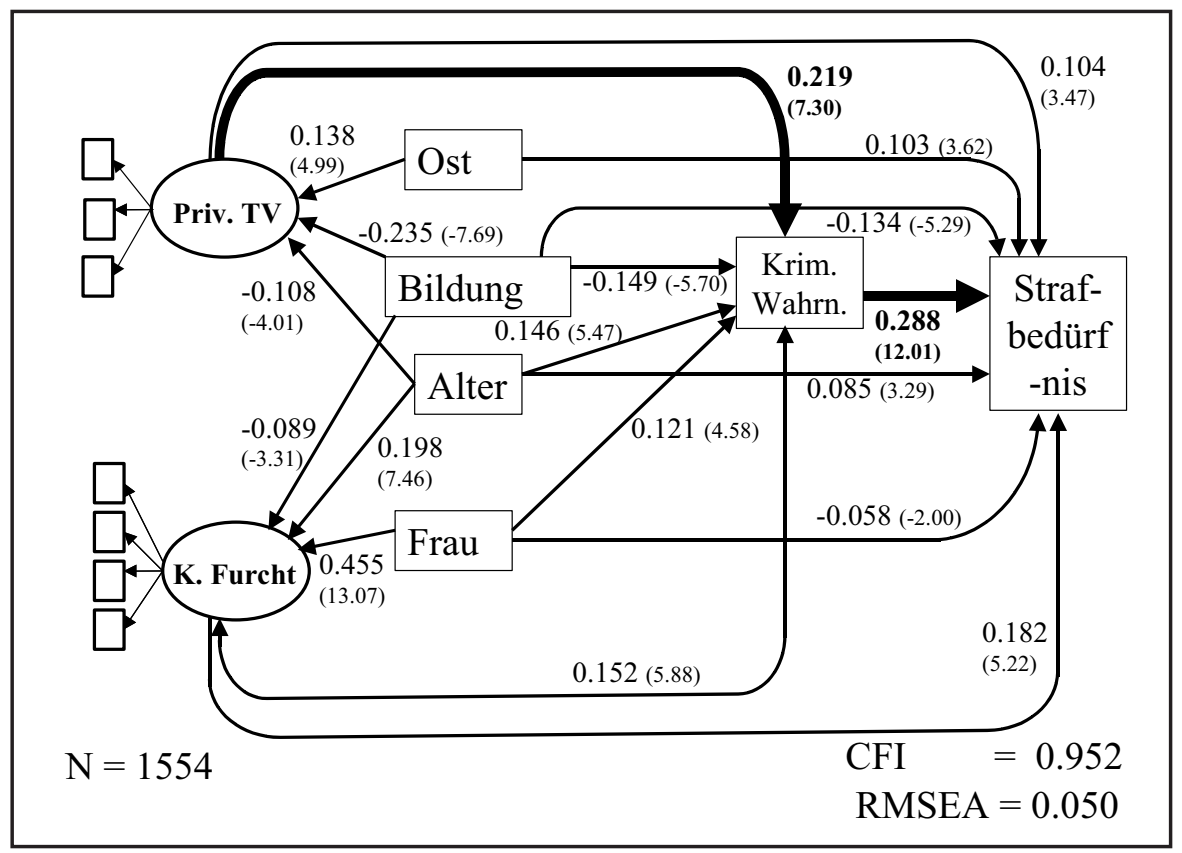

Quelle: KFN-Befragung zu Kriminalität und Strafen 2004, eigene Berechnungen, standardisierte Koeffizienten, t-Werte in Klammern

Auch die zu Beginn formulierte Hypothese, dass eine durch Mediennutzung bedingte verzerrte Sicht auf Kriminalität das Bedürfnis nach härteren Strafen fördert, unterstellt somit eine komplexere Kausalstruktur. In Abbildung 5 ist darum ein Pfadmodell mit ordinalen abhängigen Variablen dargestellt, das sowohl den selektiven Zugang zu privaten Nachrichtensendungen kontrolliert als auch den über die verzerrte Kriminalitätswahrnehmung vermittelten Effekt privater Nachrichtensendungen auf die Einstellung zum Strafen abbildet. Die im Folgenden besonders interessierenden standardisierten Einflussfaktoren, die in ihrer Einflussstärke miteinander verglichen werden können, sind durch fett gedruckte Pfeile dargestellt. 
Alle im Modell in Abbildung 5 dargestellten Effekte sind signifikant. Mit einem comparative fit index (CFI) von 0.952 sowie einem root mean square error of approximation (RMSEA) von 0.050 ist das Modell den Daten gut angepasst (Cin-Yun 2002, S.15f). Zudem wurden die Befunde zu den eingangs formulierten Hypothesen 2 und 3 durch eine Kreuzvalidierung erhärtet (vgl. Anhang).

In Abbildung 5 ist zu sehen, dass die Nutzung privater Nachrichtensendungen auch auf direktem Wege das Verlangen nach härteren Strafen erhöht. Jedoch zielte die eigentliche Hypothese darauf ab, dass Kriminalität aufgrund des hohen Nachrichtenfaktors in den privaten Medien eine übermäßige Präsenz erfährt, die auf der kognitiven Ebene beim Rezipienten, der ja selbst wiederum besonders sensibilisiert ist für Kriminalitätsberichte, zu falschen Vorstellungen über die Kriminalitätsentwicklung führt. Erst wenn sich diese Vorstellung ausgebildet hat, ist es folgerichtig, alltagsweltliches Wissen über den abschreckenden Effekt von Strafen zu mobilisieren und anzunehmen, härtere Strafen könnten dem vermeintlichen Trend entgegenwirken. Der Effekt der Nutzung privater Nachrichtensendungen auf das Strafbedürfnis sollte also durch die vermutete Kriminalitätsentwicklung vermittelt sein.

Im Pfadmodell zeigt sich des Weiteren der sozial selektive Zugang zu privaten Nachrichtensendungen. Mit steigendem Alter, stärker noch mit zunehmendem Bildungsniveau, geht die Nutzung von kriminalitätshaltigen privaten Nachrichtensendungen zurück. Auffällig ist, dass diese im Osten Deutschlands häufiger gesehen werden als im Westen. Ein hohes Bildungsniveau reduziert zudem die Kriminalitätsfurcht, jedoch nimmt diese mit steigendem Alter zu und ist außerdem bei Frauen deutlich größer als bei Männern.

Der vermutete Kriminalitätsanstieg zwischen 1993 und 2003 wird von Älteren sowie von Frauen höher eingeschätzt, während ein höherer formaler Bildungsgrad zu einer realistischeren Einschätzung beiträgt. Wichtig ist nun der deutliche Effekt der Nutzungsskala »Privat $\mathrm{TV}$ « auf die wahrgenommene Kriminalitätsentwicklung. Demnach ist auch Hypothese 2 aufrechtzuerhalten: Auch im Pfadmodell zeigt sich, dass kriminalitätshaltige Nachrichtensendungen des Privatfernsehens zu einem verzerrten Bild der Kriminalität führen. Je häufiger Nachrichtensendungen des Privatfernsehens genutzt werden, desto stärker ist der vermutete Anstieg der Delikte. Mit der Kriminalitätsfurcht steht dieses Bild ebenfalls in Verbindung. Allerdings ist die Kausalität unbestimmt, denn möglicherweise führt entweder die Furcht dazu, dass man die Kriminalitätsentwicklung überschätzt - oder aber man überschätzt die Kriminalität und zieht daraus konsequenterweise den Schluss, dass dies als Anlass zu Kriminalitätsfurcht bzw. zu Vorsichtsmaßnahmen zu werten sei. Aus diesem Grund wurde anstelle eines kausalen ein korrelativer Zusammenhang unterstellt, der erwartungsgemäß positiv ist.

Schließlich wird die Einstellung zum Strafen bzw. das Strafbedürfnis von allen im Modell berücksichtigten Variablen beeinflusst. Personen mit höherer Bildung und Frauen tendieren weniger zu der Auffassung, dass die üblicherweise verhängten Strafen zu gering seien. Abgesehen davon ist hervorzuheben, dass Ältere sowie Personen mit höherer Kriminalitätsfurcht ein größeres Strafbedürfnis aufweisen und vor allem, wie nach Hypothese 3 zu erwarten war, dass auch die Fehleinschätzung über die Kriminalitätsentwicklung einen direkten positiven Effekt zeigt. Als ein gegenüber der Kriminalitätsfurcht sogar noch stärkerer Effekt erhöht das Ausmaß, in dem die Befragten eine höhere Zahl von Delikten in Jahre 2003 annehmen, das Strafbedürfnis.

\section{Medienwirkungen durch Kultivation und Selektion}

Auch Massenmedien sind Beobachter, die aktiv Unterscheidungen treffen und selbst bestimmen, über welche Seite der Unterscheidung sie kommunizieren. Informieren sie über Kriminalität oder andere Skandale, setzen sie zugleich die Möglichkeit normkonformen Verhaltens voraus. Sie problematisieren jedoch in der Regel nicht, warum ihrer Beobachtung gerade die 
Unterscheidung skandalös/nichtskandalös zugrunde liegt und nicht ein anderes Schema (Luhmann 1996, S.207). Diese Problematisierung ist Aufgabe der Wissenschaft. Die Befunde dieser empirischen Studie setzen vor allem bei den Rezipienten an und weisen darauf hin, dass die Selektion von Berichtenswertem, die durch die Medien selbst anhand der eigenen Schemata getroffen wird, nicht ohne Folgen für die Rezipienten bleibt. In Nachrichtensendungen des Privatfernsehens, die sich seit den frühen 1990er Jahren in der deutschen Medienlandschaft etabliert haben, wird im Vergleich $\mathrm{zu}$ öffentlich-rechtlichen Nachrichtensendungen deutlich mehr über Kriminalität berichtet. Zwar erlaubt der hier für Nachrichtensendungen gezeigte Trend für die Jahre 2000 bis einschließlich 2003 keine Aussagen über eine allgemeine Zunahme der Kriminalitätsberichte. Zumindest seit Ende 2001 deutet sich sogar eher eine Abnahme an, was möglicherweise durch eine Verschiebung des Themenschwerpunktes hin zum internationalen Terrorismus und Krieg begründet sein könnte. Aber mit der Etablierung privater Sender seit den frühen 1990er Jahren sind Nachrichtenformate entstanden, welche aus ökonomischen Gründen gezwungen sind, die Aufmerksamkeit der Rezipienten sicherzustellen. Daher überrascht es nicht, dass nach den Befunden dieser Arbeit zumindest in der untersuchten Periode in privaten Nachrichtensendungen ungefähr doppelt so häufig über das reizgeladene Thema der Kriminalität berichtet wird wie in öffentlich-rechtlichen Nachrichtensendungen. Auf der Mikroebene der Rezipienten hinterlässt diese Kommunikation Spuren, denn vor allem jene Personen, die häufig private Fernsehnachrichten sehen, vermuten fälschlicherweise einen Anstieg der Kriminalität. Darüber hinaus wurde deutlich, dass die unter anderem durch Einfluss privater Nachrichtensendungen erzeugte Fehleinschätzung eine rigidere Einstellung zum Strafen und ein Bedürfnis nach härteren Strafen fördert. Ermittelt wurde dieser Befund im Rahmen eines Pfadmodells, in dem zumindest einige wesentliche Einflussvariablen berücksichtigt wurden, die mit der sozial sehr selektiven Nutzung privater Nachrichtensendungen in Verbindung stehen. Somit ist die Vorgehensweise dieser Arbeit auch von medienwissenschaftlichem Interesse, weil versucht wurde, einige Unzulänglichkeiten der empirischen Analysen von Gerbner zu umgehen. Gerbners Theorie der Kultivation ist trotz vieler Einwände gegen seine empirischen Untersuchungen sehr etabliert. Möglicherweise trägt die vorliegende Arbeit dazu bei, dass künftige Forschung, die an Gerbners Ansatz anknüpft, noch stärker die Determinanten der Auswahl von Medieninhalten in der Vordergrund stellt, wie es im Uses-andGratifications-Ansatz betont wird. Die Annahme komplexer Kausalstrukturen ist dabei unerlässlich. Gleichwohl handelt es sich gerade bei auf Querschnittsdaten basierenden Analysen nur um Modelle, die, wie gut auch immer sie den Daten angepasst sind, an die Wirklichkeit herangetragen werden. Immerhin haben die theoretische Diskussion und der Verweis auf bisherige Befunde gezeigt, dass die festgestellten Einflusskonstellationen überaus plausibel sind. Wünschenswert wären künftig Längsschnittuntersuchungen, die über längere Zeiträume hinweg Mediennutzung, Kriminalitätswahrnehmung und Strafbedürfnis erfassen und direkt mit Zeitreihen der realen Kriminalitätsentwicklung verknüpfen. $\mathrm{Zu}$ bedenken ist aber, dass mittels Paneldaten, die nur kurze Zeiträume erfassen, wie dies etwa in der Studie von Reuband (1998) der Fall ist, das Problem der kausalen Interpretation des Effekts von Mediennutzung auf Kriminalitätsfurcht oder wahrgenommene Kriminalitätsentwicklung nicht zu lösen ist. Allein die temporale Anordnung der Variablen reicht für eine eindeutige Erklärung der Ursache-Wirkungsbeziehungen nicht aus, wenn die erklärende Variable (hier die Nutzungshäufigkeit von Nachrichtensendungen) über den Zeitverlauf sehr stabil ist. So berichtete Reuband (1998, S.141) über drei Wellen Pfadkoeffizienten um 0.80, was den Schluss nahe legt, dass zu allen drei Zeitpunkten unbeobachtete Faktoren in nahezu derselben Weise die Nutzungshäufigkeit der Nachrichtensendungen beeinflussen. Werden Paneldaten zur Mediennutzung und Kriminalitätswahrnehmung aber über längere Zeiträume erhoben, könnten Prozesse der »Sozialisation « der Rezipienten in ein jeweiliges Muster der Mediennutzung untersucht und erst dadurch die kausale Struktur der Einflüsse aus den Daten selbst abgele- 
sen werden. Weil solche Daten aber bislang in Deutschland nicht existieren und auch überaus schwer zu erheben sind, bleibt die Forschung auf theoretisch zu begründende Annahmen angewiesen.

Immerhin legen unsere Befunde Schlussfolgerungen über kriminalpolitische Konsequenzen aus der medialen Thematisierung von Kriminalität nahe. Zweifelsohne ist es plausibel, bei jenen Personen, die intensiv und häufig privaten Nachrichtensendungen ausgesetzt sind und die einen vergleichsweise großen Teil ihrer Information über Kriminalität aus diesen Sendungen beziehen, eine Anfälligkeit für populistische Kriminalpolitik zu vermuten. Geht man von individualisierten sowie von zunehmend personen- und themenabhängigen Wahlkämpfen aus, ist zu erwarten, dass Kriminalität als ein Thema erkannt wird, mit dem politische Differenzierungen hergestellt werden können. Erste Überlegungen in diese Richtung haben wir in Pfeiffer, Windzio und Kleimann (2004) angestellt. In welcher Weise das verzerrte Bild der Kriminalitätsentwicklung kriminalpolitische Folgen hat, und wie beispielsweise die in den letzten zehn Jahren durchgesetzten Strafrechtsverschärfungen mit einer übermäßigen Präsenz der Kriminalität in den Medien in Verbindung stehen, wird derzeit am Kriminologischen Forschungsinstitut Niedersachsen ausführlich untersucht.

\section{Anhang}

\section{Frageformulierungen:}

Mediennutzung:

Im Fernsehen gibt es ein großes Angebot an Sendungen, in denen es auch um Kriminalität geht. Im Folgenden stehen einige Arten von Sendungen mit Beispielen in Klammern. Wie häufig schauen Sie sich solche Sendungen im Fernsehen an? (BITTE MACHEN SIE IN jeder Zeile ein Kreuz!)

$\begin{array}{lllllll} & \begin{array}{l}\text { (Fast) } \\ \text { täglich }\end{array} & \begin{array}{l}\text { Mehrmals } \\ \text { in der } \\ \text { Woche }\end{array} & \begin{array}{l}\text { Einmal in } \\ \text { der Woche }\end{array} & \begin{array}{l}\text { Mehrmals } \\ \text { im Monat }\end{array} & \begin{array}{l}\text { Einmal im Nie } \\ \text { Monat und } \\ \text { seltener }\end{array} \\ \text { Nachrichtensendungen: } & & & & & & \\ \text { Tagesschau / Tagesthemen.. } & \square & \square & \square & \square & \square & \square \\ \text { heute / heute journal.. } & \square & \square & \square & \square & \square & \square \\ \text { RTL Aktuell .. } & \square & \square & \square & \square & \square & \square \\ \text { 18.30 - Sat.1-Nachrichten.. } & \square & \square & \square & \square & \square & \square \\ \text { Pro 7 Nachrichten.. } & \square & \square & \square & \square & \square & \square\end{array}$

Kriminalitätswahrnehmung:

Unten werden verschiedene Arten von Straftaten genannt. Bitte geben Sie jeweils an, ob solche Taten nach Ihrem Eindruck in Deutschland zwischen 1993 und 2003 häufiger geworden, gleich häufig geblieben oder seltener geworden sind. (BITTE BEANTWORTEN SIE DIE FRAGE FÜR JEDE DER STRAFTATEN, MACHEN SIE ALSO IN jeder Zeile ein Kreuz!)

Solche Straftaten sind nach meinem Eindruck in den letzten 10 Jahren...

\begin{tabular}{|c|c|c|c|c|c|c|c|}
\hline+ & $\begin{array}{l}\text { sehr viel } \\
\text { häufiger } \\
\text { geworden }\end{array}$ & $\begin{array}{l}\text { viel } \\
\text { häufiger } \\
\text { geworden }\end{array}$ & $\begin{array}{l}\text { etwas } \\
\text { häufiger } \\
\text { geworden }\end{array}$ & $\begin{array}{l}\text { gleich } \\
\text { geblieben }\end{array}$ & $\begin{array}{l}\text { etwas } \\
\text { seltener } \\
\text { geworden }\end{array}$ & $\begin{array}{l}\text { viel } \\
\text { seltener } \\
\text { geworden }\end{array}$ & $\begin{array}{l}\text { sehr viel } \\
\text { seltener } \\
\text { geworden }\end{array}$ \\
\hline & 3 & 2 & 1 & 0 & -1 & -2 & -3 \\
\hline Alle Straftaten & $\square$ & $\square$ & $\square$ & $\square$ & $\square$ & $\square$ & $\square$ \\
\hline
\end{tabular}

insgesamt... 
Strafbedürfnis

Welchen Eindruck haben Sie von den Strafen, die von deutschen Gerichten verhängt werden?

Sie können Ihre Meinung zwischen »viel zu gering« $(-3)$, »angemessen« $(0)$ und »viel zu hoch $\ll(+3)$ abstufen.

(BITTE MACHEN SiE JEWEILS EIN KREUZ PRO FRAGE.)

Schaut man sich die Schwere der Straftaten insgesamt an, erscheinen mir die verhängten Strafen im Allgemeinen ...

Viel zu gering

Angemessen

Viel zu hoch

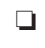

$-\mathbf{3}$

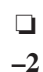

$-2$

$\square$
-1

$\square$

1

$\square$

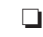

3

Tabelle A1: Mittelwerte und Standardabweichungen der unabhängigen Variablen

\begin{tabular}{lccccc}
\hline & $\mathrm{N}$ & mean & stddev & min & max \\
\hline Frau (=1, sonst 0) & 1554 & 0.52 & 0.49 & 0 & 1 \\
Ausbildungsjahre & 1554 & 11.30 & 3.69 & 0 & 19 \\
Alter & 1554 & 46.11 & 16.22 & 15 & 85 \\
Ostdeutschland & 1554 & 0.21 & 0.41 & 0 & 1 \\
Nutzung privater Nachrichtensendungen & 1554 & 43.70 & 32.04 & 0 & 100 \\
Kriminalitätsfurcht: Vorsichtsmaßnahmen & 1554 & 45.65 & 26.34 & 0 & 100 \\
\hline
\end{tabular}

Tabelle A1 zeigt die Mittelwerte und Standardabweichungen der unabhängigen Variablen. Aufgrund der stark rechtsschiefen Verteilung (nur 8 von 1554 Personen waren der Auffassung, die verhängten Strafen seinen zu hoch) mussten die Kategorien 0 bis +3 des Strafbedürfnisses zusammengefasst werden, so dass sich für die ordinalen abhängigen Variablen des Strafbedürfnisses und der Kriminalitätswahrnehmung folgende Häufigkeiten ergaben:

Tabelle A2: Häufigkeitsverteilung des Strafbedürfnisses, $\mathrm{N}=1554$

\begin{tabular}{l|l}
\hline $\begin{array}{l}\text {..erscheinen mir die verhängten } \\
\text { Strafen im Allgemeinen... }\end{array}$ & N \\
\hline 1 angemessen/(viel) zu hoch & 133 \\
2 & 358 \\
3 & 470 \\
4 viel zu gering & 593 \\
& \\
\hline
\end{tabular}

Tabelle A3: Häufigkeitsverteilung der Kriminalitätswahrnehmung, $\mathrm{N}=1554$

\begin{tabular}{l|l}
\hline $\begin{array}{l}\text {...sind nach meinem Eindruck in } \\
\text { den letzten } 10 \text { Jahren ... }\end{array}$ & $\mathrm{N}$ \\
\hline 1 etwas/(sehr) viel seltener & 32 \\
2 gleich geblieben & 100 \\
3 etwas häufiger & 399 \\
4 viel häufiger & 617 \\
5 sehr viel häufiger & 406 \\
\hline
\end{tabular}

Quelle: KFN-Befragung zu Kriminalität und Strafen 2004 eigene Berechnungen

Das Pfadmodell wurde anhand einer Kreuzvalidierung überprüft. Dabei wurde zunächst ein Subsample zur Schätzung der Effekte (»calibration sample«) von 25\% gezogen und anhand der drei übrigen $25 \%$ Stichproben validiert (»validation samples«). Insgesamt sind die Befunde sehr ähnlich. Erwähnenswert ist der direkte Effekt der Mediennutzung auf das 
Strafbedürfnis, der nicht immer signifikant ist. Es wurde im theoretischen Teil auch kein direkter Effekt vermutet, sondern nur ein indirekter, über die Mediennutzung vermittelter Effekt.

Tabelle A3: Die zentralen Effekte des Pfadmodells nach Kreuzvalidierung, standardisierte Koeffizienten, T-Statistiken in Klammern

\begin{tabular}{|l|l|l|l|l|}
\hline & Calibration & Validation 1, & Validation 2, & Validation 3, \\
& N=407, & N=400, & N=404, & N=343, \\
& CFI=0.959 & CFI=0.933 & CFI=0.938 & CFI=0.958 \\
& RMSEA & RMSEA= & RMSEA= & RMSEA= \\
& 0.044 & 0.058 & 0.054 & 0.044 \\
\hline Kriminalitätswahrn. $\leftarrow$ Privat TV & 0.179 & $0.211(3.793)$ & $0.203(3.541)$ & $0.292(4.198)$ \\
Strafbedürfnis $\leftarrow$ Privat TV & $(3.056)$ & $0.075(1.270)$ & $0.135(2.430)$ & $0.146(2.485)$ \\
Strafbedürfnis $\leftarrow$ Kriminalitätswahrn & 0.080 & $0.267(5.750)$ & $0.365(8.164)$ & $0.221(4.658)$ \\
& $(1.280)$ & & & \\
& 0.281 & & & \\
& $(5.781)$ & & & \\
\hline
\end{tabular}

\section{Literatur}

Bonfadelli, H. (2004a): Medienwirkungsforschung I. Grundlagen. Konstanz: UVK.

Bonfadelli, H. (2004b): Medienwirkungsforschung II. Anwendungen. Konstanz: UVK.

Carroll, G. R. (1984): Publish and perish. The organizational ecology of newspaper industries. London: JAI Press.

Cavender, G. (2004): Media and Crime Policy. A Reconsideration of David Garland's The Culture of Control, in: Punishment \& Society 6, S.335-348.

Ching-Yun, Y. (2002): Evaluating Cutoff Criteria of Model Fit Indices for Latent Variable Models with Binary and Continuous Outcomes. Dissertation, University of California, Los Angeles 2002.

Derwein, Ch. (1995). Wie wird Kriminalität in der Presse dargestellt, ist die Darstellung wirklichkeitsfremd, und gibt es Entsprechungen im Vorstellungsbild der Bevölkerung. Rechtswissenschaftliche Dissertation, Johann-Wolfgang-Goethe-Universität, Frankfurt a.M.

Ditton, J./ Chadee, D./ Farrall, St./ Gilchrist, E./ Bannister, J. (2004): From Imitation to Intimidation. A Note on the Curious and Changing Relationship between the Media, Crime and Fear of Crime, in: British Journal of Criminology 44, S.595-610.

Eschholz, S. (1997): The Media and Fear of Crime: A Survey of the Research, in: Journal of Law and Public Policy 9, S.37-59.

Hirsch, P. M. (1980): The Scary World of the Nonviewer and Other Anomalies. A Reanalysis of Gerbner et al.'s Findings on Cultivation Analysis Part I, in: Communication Research 7, S.403- 456.

Hirsch, P. M. (1981): On Not Learning From One's Own Mistakes. A Reanalysis of Gerbner et al.'s Findings on Cultivation Analysis Part II, in: Communication Research 8, S.3-37.

Leonhardt, U. (1990): Mord ist ihr Beruf. Eine Geschichte des Kriminalromans. München: CH Beck.

Long, J. S. (1997): Regression Models for Categorical and Limited Dependent Variables. London: Sage.

Long, J. S./ Freese, J. (2004): Regression Models for Categorical Dependent Variables using STATA. College Station, Texas: Stata Press.

Luhmann, N. (1984): Soziale Systeme. Grundriss einer allgemeinen Theorie. Frankfurt/M.: Suhrkamp.

Luhmann, N. (1996): Die Realität der Massenmedien. 3. Aufl., Wiesbaden: VS Verlag.

McQuail, D. (1984): With the Benefit of Hinsight: Reflections on Uses and Gratifications Research, in: Critical Studies in Mass Communication 1, S.177-193. 
MedienTenor (2004): Forschungsbericht Nr. 142. Bonn.

Meyer, P. (1982): Soziologie und Soziobiologie. Eine Einführung in die biologischen Voraussetzungen sozialen Handelns. Darmstadt: Luchterhand.

Muthén, L. K./ Muthén B. O. (1998-2004): Mplus®. User’s guide. Statistical models with latent variables. Third edition. Los Angeles, CA: Muthén \& Muthén.

Neisser, U. (1979): Kognition und Wirklichkeit. Prinzipien und Implikationen der kognitiven Psychologie. Stuttgart: Klett-Kotta.

Niedersächsisches Ministerium für Inneres und Sport (2005): Polizeiliche Kriminalstatistik in Niedersachsen 2004. Hannover.

Peltu, M. (1985): The Role of Comunication Media, in: H. Ottway/M. Peltu (eds.): Regulating Industrial Risks: Science, Hazards and Public Protection, London 1985, S. 128-148.

Pfeiffer, Ch./ Windzio M./ Kleimann, M. (2004): Die Medien, das Böse und wir. Zu den Auswirkungen der Mediennutzung auf Kriminalitätswahrnehmung, Strafbedürfnisse und Kriminalpolitik, in: Monatsschrift für Kriminologie und Strafrechtsreform 87, S.415-435.

Plake, K. (2004): Handbuch Fernsehforschung. Befunde und Perspektiven. Wiesbaden: VS-Verlag.

Reuband, K.-H. (1998): Kriminalität in den Medien. Erscheinungsformen, Nutzungsstruktur und Auswirkungen auf die Kriminalitätsfurcht, in: Soziale Probleme 9, S.125-153.

Riedl, R. (1988): Biologie der Erkenntnis. Die stammesgeschichtlichen Grundlagen der Vernunft. München: DTV.

Roberts, J. V. (1992): Public Opinion, Crime, and Criminal Justice, in: Crime and Justice 16, S.99-180.

Rubin, A. (2002): The Uses-and-Gratifications Perspective, in: J. Bryant / D. Zillmann (Hrsg.): Perspectives on Media Effects. Mahwah, N.J.: Erlbaum, S.525-548.

Scharf, W./ Mühlenfeld H.-U./ Stockmann, R. (1999): Zur Kriminalitätsberichterstattung in der Presse, in: Publizistik 44, S.445-462.

Signorielli, N./ Morgan, M. (1996): Cultivation Analysis. New Directions in Media Effects Research. Newbury Park: Sage.

STATA Corporation (2003): Cross-Sectional Time-Series. Reference Manual Vers. 8. College Station, Texas: STATA Press.

Michael Windzio, Matthias Kleimann, Kriminologisches Forschungsinstitut Niedersachsen,

Lützerodestraße 9, 30161 Hannover mwindzio@kfn.uni-hannover.de.0511-34836-32 mkleimann@kfn.uni-hannover.de.0511-34836-14 